\title{
Positive Fixed Point of Strict Set Contraction Operators on Ordered Banach Spaces and Applications
}

\author{
Meiqiang Feng, ${ }^{1}$ Xuemei Zhang, ${ }^{2,3}$ and Weigao $\mathrm{Ge}^{3}$ \\ ${ }^{1}$ School of Applied Science, Beijing Information Science E Technology University, Beijing 100192, China \\ ${ }^{2}$ Department of Mathematics and Physics, North China Electric Power University, Beijing 102206, China \\ ${ }^{3}$ Department of Mathematics, Beijing Institute of Technology, Beijing 100081, China
}

Correspondence should be addressed to Meiqiang Feng, meiqiangfeng@sina.com

Received 4 February 2010; Revised 8 May 2010; Accepted 7 July 2010

Academic Editor: Ferhan M. Atici

Copyright (c) 2010 Meiqiang Feng et al. This is an open access article distributed under the Creative Commons Attribution License, which permits unrestricted use, distribution, and reproduction in any medium, provided the original work is properly cited.

The fixed point theorem of cone expansion and compression of norm type for a strict set contraction operator is generalized by replacing the norms with a convex functional satisfying certain conditions. We then show how to apply our theorem to prove the existence of a positive solution to a second-order differential equation with integral boundary conditions in an ordered Banach space. An example is worked out to demonstrate the main results.

\section{Introduction}

The theory of integral and differential equations in Banach spaces, as two new branches of nonlinear functional analysis, has developed for no more than forty years, but it has extensive applications in such domains as the critical point theory, the theory of partial differential equations, and eigenvalue problems. For an introduction of the basic theory of integral and differential equations in Banach spaces, see Guo et al. [1], Guo and Lakshmikantham [2], Lakshmikantham and Leela [3], and Demling [4], and the references therein. In recent years, the theory of integral and differential equations in Banach spaces has become an important area of investigation in both pure and applied mathematics (see, for instance, [5-18] and references cited therein).

On the other hand, the theory of fixed point is an important tool to study various boundary value problems of ordinary differential equations, difference differential equations, and dynamic equations on time scales. An overview of such results can be found in Guo et al. [1], in Guo and Lakshmikantham [2], and in Demling [4]. The Krasnoselskii's fixed 
point theorem concerning cone compression and expansion of norm type is worth mentioing here as follows (see $[1,2,4]$ ).

Theorem 1.1. Let $\Omega_{1}$ and $\Omega_{2}$ be two bounded open sets in Banach space $E$, such that $0 \in \Omega_{1}$ and $\bar{\Omega}_{1} \subset \Omega_{2}$. Let $P$ be a cone in $E$ and let operator $A: P \cap\left(\bar{\Omega}_{2} \backslash \Omega_{1}\right) \rightarrow P$ be completely continuous. Suppose that one of the following two conditions is satisfied:

(a) $\|A x\| \geq\|x\|, \forall x \in P \cap \partial \Omega_{1}$, and $\|A x\| \leq\|x\|, \forall x \in P \cap \partial \Omega_{2} ;$

(b) $\|A x\| \leq\|x\|, \forall x \in P \cap \partial \Omega_{1}$ and $\|A x\| \geq\|x\|, \forall x \in P \cap \partial \Omega_{2}$.

Then, $A$ has at least one fixed point in $P \cap\left(\Omega_{2} \backslash \bar{\Omega}_{1}\right)$.

To generalize Theorem 1.1, one may consider the weakening of one or more of the following hypotheses: (i) the operator $A$, (ii) the norm.

In [19], Sun generalized Theorem 1.1 for completely continuous operator to strict set contraction operator and obtain the following results.

Theorem 1.2. Let $K$ be a cone of Banach space $E$ and $K_{r}=\{x \in K:\|x\| \leq r\}, K_{r, R}=\{x \in K, r \leq$ $\|x\| \leq R\}$ with $R>r>0$. Suppose that $A: K_{R} \rightarrow K$ is a strict set contraction such that one of the following two conditions is satisfied:

(a) $\|A x\| \geq\|x\|, \forall x \in K,\|x\|=r ;\|A x\| \leq\|x\|, \forall x \in K,\|x\|=R$;

(b) $\|A x\| \leq\|x\|, \forall x \in K,\|x\|=r ;\|A x\| \geq\|x\|, \forall x \in K,\|x\|=R$.

Then, $A$ has a fixed point $x \in K_{r, R}$.

Recently, in [20], Anderson and Avery generalized the fixed point theorem of cone expansion and compression of norm type by replacing the norms with two functionals satisfying certain conditions to produce a fixed point theorem of cone expansion and compression of functional type. In [21], Guo and Ge extended Krasnoselskii's fixed point theorem by choosing two functionals that satisfy certain conditions which are used in place of the norm. In [22], Zhang and Sun generalized the classical Krasnoselskii's fixed point theorem concerning cone compression and expansion of norm type. The interesting point is that they took place norm by convex functional.

In the past few years, we also notice a class of boundary value problems with integral boundary conditions appeared in heat conduction, chemical engineering, underground water flow, thermo elasticity, and plasma physics. Such problems include two, three, multi point and nonlocal boundary value problems as special cases and attracted the attention of Gallardo [23], Karakostas and Tsamatos [24], Lomtatidze and Malaguti [25], and others included in the references therein. On the other hand, we refer the reader to papers by Ahmad et al. [26], Feng et al. [27], Boucherif [28], Infante and Webb [29], Kang et al. [30], Ma [31], Webb [32], Webb and Infante [33, 34], Yang [35], Zhang et al. [36-38], and Chang et al. [39] for other recent results on nonlinear boundary value problems with integral boundary conditions.

Motivated by works mentioned above, we intend in this paper to generalize the fixed point theorem of cone expansion and compression of norm type for strict set contraction operator. The generalization allows the user to choose a convex functional that satisfies certain conditions which are used in place of the norm. In applications to boundary value problems, the functional will typically be maximum of the function over a specific interval. The flexibility of using functionals instead of norms allows the theorem to be used in a 
wider variety of situations. Our results either improve or generalize the corresponding results due to [19-22] and many of others. As an application of our main results, we consider the existence of positive solutions for second-order differential equations with integral boundary conditions in an ordered Banach space. On the other hand, our conditions are weaker than those of [22].

The organization of this paper is as follows. We will introduce some lemmas and notations in the rest of this section. In Section 2, the main results will be stated and proved. In Section 3, as an application of our main results, the existence of positive solutions for a second-order boundary value problem with integral boundary conditions in ordered Banach spaces is considered. Finally, in Section 4, one example is also included to illustrate the main results.

Basic facts about ordered Banach space $E$ can be found in [1-4]. Here we just recall a few of them. The cone $P$ in $E$ induces a partial order on $E$, that is, $x \leq y$ if and only if $y-x \in P . P$ is said to be normal if there exists a positive constant $N$ such that $\theta \leq x \leq y$ implies $\|x\| \leq N\|y\|$. Without loss of generality, suppose, in the present paper, the normal constant $N=1$.

For a bounded set $V$ in Banach space $E$, we denote $\alpha(V)$ the Kuratowski measure of noncompactness (see [1-4], for further understanding). The operator $A: D \rightarrow E(D \subset E)$ is said to be a $k$-set contraction if $A: D \rightarrow E$ is continuous and bounded and there is a constant $k \geq 0$ such that $\alpha(A(S)) \leq k \alpha(S)$ for any bounded $S \subset D$; a $k$-set contraction with $k<1$ is called a strict set contraction.

In the following, denote the Kuratowski's measure of noncompactness by $\alpha(\cdot)$.

For the application in the sequel, we first state the following definition and lemmas which can be found in [1], and some notation.

Definition 1.3. Let $S$ be a bounded set of a real Banach space $E$. Let $\alpha(S)=\inf \{\delta>0: S$ be expressed as the union of a finite number of sets such that the diameter of each set does not exceed $\delta$, that is, $S=\bigcup_{i=1}^{m} S_{i}$ with $\left.\operatorname{diam}\left(S_{i}\right) \leq \delta, i=1,2, \ldots, m\right\}$. Clearly, $0 \leq \alpha(S)<\infty . \alpha(S)$ is called the Kuratowski's measure of noncompactness.

Definition 1.4. Let $P$ be a cone of a real Banach space $E$. If $P^{*}=\left\{\Psi \in E^{*} \mid \Psi(x) \geq 0, \forall x \in P\right\}$, then $P^{*}$ is a dual cone of cone $P$.

Definition 1.5. Let $P$ be a cone of real Banach space $E . \rho: P \rightarrow R$ is said to be a convex functional on $P$ if $\rho(t x+(1-t) y) \leq t \rho(x)+(1-t) \rho(y)$ for all $x, y \in P$ and $t \in[0,1]$.

Definition 1.6. A subset $X \subset E$ is said to be a retract of $E$ if there exists a continuous mapping $r: E \rightarrow X$ satisfying $r(x)=x, x \in X$.

Lemma 1.7. Let $D \subset E, D$ be a bounded set and $f$ uniformly continuous and bounded from $J \times S$ into $E$; then

$$
\alpha(f(J \times S))=\max _{t \in J} \alpha(f(t, S)), \quad \forall S \subset D
$$

\section{Main Results}

Lemma 2.1 (see [22]). Let $P$ be a cone in a real Banach space E. If $\rho: P \rightarrow[0, \infty)$ is a uniformly continuous convex functional with $\rho(\theta)=0$ and $\rho(x)>0$ for $x \neq \theta$, then $\forall r>0, D_{r}=\{x \in P$ : $\rho(x) \geq r\}$ is a retract of $E$. 
Lemma 2.2. Let $E$ be a real Banach space, $\|\cdot\|$ the norm in $E, P$ a cone in $E$, and $\Omega=\{x \in E:\|x\|<$ $R\}$, where $R$ is a positive real number. Suppose that $A: P \cap \bar{\Omega} \rightarrow P$ is a $k$-set contraction with $k<1$ and $\rho: P \rightarrow[0, \infty)$ is a uniformly continuous convex functional with $\rho(\theta)=0, \rho(x)>0, \forall x \neq \theta$ and $\rho(x) \leq\|x\|$. If

(i) $\inf _{x \in P \cap \partial \Omega} \rho(x)>0$, and there exists $\delta>0$ such that $R / \inf _{x \in P \cap \partial \Omega} \rho(x) \leq 1+\delta / k$ and $\rho(A x) \geq(k+\delta) \rho(x), \forall x \in P \cap \partial \Omega$;

(ii) $A x \neq \mu x, \mu \in(0,1], \forall x \in P \cap \partial \Omega$,

hold, then the fixed point index $i(A, P \cap \Omega, P)=0$.

Proof. Without loss of generality, we suppose $k+\delta<1$ (If $k+\delta>1$, then let $N=k+\delta$. The proof is the same as the following process). Let $N=1 /(k+\delta)$, then $N A$ is a strict set contraction. Considering $h_{t}(x)=t A x+(1-t) N A x, t \in[0,1], x \in P \cap \partial \Omega$. If there exists $t_{0} \in[0,1], x_{0} \in P \cap \partial \Omega$ such that $x_{0}=t_{0} A x_{0}+\left(1-t_{0}\right) N A x_{0}$, then $A x_{0}=\left(1 /\left(t_{0}+N\left(1-t_{0}\right)\right)\right) x_{0}$, which contradicts with (ii). Then by the homotopy invariance property of fixed point index, we have $i(N A, P \cap \Omega, P)=i(A, P \cap \Omega, P)$.

Let $r=\inf _{x \in P \cap \partial \Omega} \rho(x)$. Define $D_{r}=\{x \in P \mid \rho(x) \geq r\}$. It follows from $\theta \notin D_{r}$ that $d \triangleq$ $\inf _{x \in D_{r}}\|x\|>0$. From the fact that $\rho(x) \leq\|x\|$, we have $r \leq d \leq R$. In fact, since $(P \cap \partial) \Omega \subset D_{r}$, we have $d \leq R$. On the other hand, for $\forall x \in D_{r}, \rho(x)=r$, combining this with $\rho(x) \leq\|x\|$, we have $r \leq\|x\|, \forall x \in D_{r}$, then $r \leq \inf _{x \in D_{r}}\|x\|=d$. Let $M=R / r$, then by (i) we obtain $M k /(k+\delta)<1$ and $M d>\sup _{x \in P \cap \bar{\Omega}}\|x\|$, and $M D_{r} \cap(P \cap \bar{\Omega})=\emptyset$, where $M D_{r}=\left\{M x \mid x \in D_{r}\right\}$.

Let $H(t, x)=(1-t) N A x+t M N A x, \forall(t, x) \in[0,1] \times P \cap \bar{\Omega}$. Then, we have

$$
\begin{aligned}
\alpha(H(t, S)) & \leq(1-t) \alpha(N A(S))+t \alpha(M N A(S)) \\
& \leq(1-t) \frac{k}{k+\delta} \alpha(S)+t \frac{M k}{k+\delta} \alpha(S) \\
& <\frac{M k}{k+\delta} \alpha(S), \quad \forall S \subset P \cap \Omega
\end{aligned}
$$

and then we obtain that $H(t, \cdot): P \cap \bar{\Omega} \rightarrow P$ is the strict set contraction. In addition, it is obvious that $H(t, x)$ is uniformly continuous about $t$ for all $x \in P \cap \bar{\Omega}$.

If there exists $x_{1} \in P \cap \partial \Omega, \quad t_{1} \in[0,1]$ such that $\left(1-t_{1}\right) N A x_{1}+t_{1} M N A x_{1}=x_{1}$, then $A x_{1}=N\left(1-t_{1}+t_{1} M\right)^{-1} x_{1}$, which contradicts with (ii). Thus by the homotopy invariance property of fixed point index, we have $i(M N A, P \cap \Omega, P)=i(N A, P \cap \Omega, P)$.

Since $D_{r}$ is a retract of $E$ by Lemma 2.1, there exists a retraction $r: E \rightarrow D_{r}$ satisfying $r(x)=x, x \in D_{r}$. Let $A_{1}=N A, \bar{A}_{1}=r \circ A_{1}$, then $\bar{A}_{1}$ is strict set contraction. From (i) and the definition of $\rho$, we have

$$
\rho\left(A_{1} x\right)=\rho(N A x) \geq N \rho(A x) \geq \rho(x) \geq r, \quad \forall x \in P \cap \partial \Omega .
$$

Therefore, $A_{1}(\partial \Omega) \subset D_{r}$, that is, $\bar{A}_{1} x=A_{1} x, \forall x \in P \cap \partial \Omega$. Then $i\left(M \bar{A}_{1}, P \cap \Omega, P\right)=i\left(M A_{1}, P \cap\right.$ $\Omega, P)$.

If $i\left(A_{1}, P \cap \Omega, P\right) \neq 0$, then $i\left(M \bar{A}_{1}, P \cap \Omega, P\right) \neq 0$, which implies that $M \bar{A}_{1}$ has a fixed point $x^{*}$ in $P \cap \Omega$. Thus $x^{*}=M \bar{A}_{1} x^{*} \in M D_{r}$. It is a paradox. The proof is complete. 
Lemma 2.3 (see [2]). Let $P$ be a cone and $\Omega$ a bounded open set in $E$ with $\theta \in \Omega$. Suppose that $A: P \cap \bar{\Omega} \rightarrow P$ is condensing and

$$
A x \neq \mu x, \quad \forall x \in P \cap \partial \Omega, \mu \geq 1 .
$$

Then $i(A, P \cap \Omega, P)=1$.

Lemma 2.4. Let $P$ be a cone and $\Omega$ a bounded open set in $E$. Suppose that $A: P \cap \bar{\Omega} \rightarrow P$ is a $k$-set contraction with $k<1$ and $\rho: P \rightarrow[0, \infty)$ is a uniformly continuous convex functional with $\rho(\theta)=0$ and $\rho(x)>0$ for $x \neq \theta$. If $\rho(A x) \leq \rho(x)$ and $A x \neq x$ for $x \in P \cap \partial \Omega$, then $i(A, P \cap \Omega, P)=1$.

Proof. If there exist $x_{1} \in P \cap \partial \Omega$ and $\mu_{1} \geq 1$ such that $A x_{1}=\mu_{1} x_{1}$, then $\mu_{1}>1$. Therefore,

$$
\rho\left(x_{1}\right)=\rho\left(\frac{1}{\mu_{1}} A x_{1}\right) \leq \frac{1}{\mu_{1}} \rho\left(A x_{1}\right) \leq \frac{1}{\mu_{1}} \rho\left(x_{1}\right)<\rho\left(x_{1}\right) .
$$

It is a paradox. From Lemma 2.3, it follows that $i(A, P \cap \Omega, P)=1$. The proof is complete.

Theorem 2.5. Let $\Omega_{1}$ be a bounded open set in $E$ such that $\theta \in \Omega_{1}$, and $\Omega_{2}=\{x \in E \mid\|x\|<R\}$ and $\bar{\Omega}_{1} \subset \Omega_{2}$. Suppose that $A: P \cap\left(\bar{\Omega}_{2} \backslash \Omega_{1}\right) \rightarrow P$ is a $k$-set contraction with $k<1$ and $\rho: P \rightarrow[0, \infty)$ is a uniformly continuous convex functional with $\rho(\theta)=0$ and $\rho(x)>0, \forall x \neq \theta$ and $\rho(x) \leq\|x\|$. If

(a) $\rho(A x) \leq \rho(x), \forall x \in P \cap \partial \Omega_{1}$;

(b) $\inf _{x \in P \cap \partial \Omega_{2}} \rho(x)>0$, and there exists $\delta>0$ such that $R / \inf _{x \in P \cap \partial \Omega_{2}} \rho(x) \leq 1+\delta / k$ and $\rho(A x) \geq(k+\delta) \rho(x)$, and $A x \neq \mu x, \mu \in(0,1], \forall x \in P \cap \partial \Omega_{2}$

hold, then $A$ has at least one fixed point in $P \cap\left(\Omega_{2} \backslash \bar{\Omega}_{1}\right)$.

Proof. It is easy to obtain the results by Lemmas 2.2 and 2.4. So we omit it.

Theorem 2.6. Let $\Omega_{1}=\{x \in E \mid\|x\|<R\}$ and $\Omega_{2}$ a bounded open set in $E$ such that $\bar{\Omega}_{1} \subset \Omega_{2}$. Suppose that $A: P \cap\left(\bar{\Omega}_{2} \backslash \Omega_{1}\right) \rightarrow P$ is a $k$-set contraction with $k<1$, and $\rho: P \rightarrow[0, \infty)$ is a uniformly continuous convex functional with $\rho(\theta)=0$ and $\rho(x)>0, \forall x \neq \theta$ and $\rho(x) \leq\|x\|$. If

(a) $\inf _{x \in P \cap \partial \Omega_{1}} \rho(x)>0$, and there exists $\delta>0$ such that $R / \inf _{x \in P \cap \partial \Omega_{1}} \rho(x) \leq 1+\delta / k$ and $\rho(A x) \geq(k+\delta) \rho(x)$, and $A x \neq \mu x, \mu \in(0,1], \forall x \in P \cap \partial \Omega_{1} ;$

(b) $\rho(A x) \leq \rho(x), \forall x \in P \cap \partial \Omega_{2}$

are satisfied, then $A$ has at least one fixed point in $P \cap\left(\Omega_{2} \backslash \bar{\Omega}_{1}\right)$.

Proof. It is easy to obtain the results by Lemmas 2.2 and 2.4. So we omit it.

Remark 2.7. If we let $k=0$, then $A$ is completely continuous. Comparing with Corollary 2.1 of [22], our conditions are weaker. 
Corollary 2.8. Let $\Omega_{1}$ be a bounded open set in E such that $\theta \in \Omega_{1}, \Omega_{2}=\{x \in E \mid\|x\|<R\}$, and $\bar{\Omega}_{1} \subset \Omega_{2}$. Suppose that $A: P \cap\left(\bar{\Omega}_{2} \backslash \Omega_{1}\right) \rightarrow P$ is a $k$-set contraction with $k<1$ and $\rho: P \rightarrow[0, \infty)$ is a uniformly continuous convex functional with $\rho(\theta)=0$ and $\rho(x)>0, \forall x \neq \theta$ and $\rho(x) \leq\|x\|$. If

(a) $\rho(A x) \leq \rho(x), \forall x \in P \cap \partial \Omega_{1}$;

(b) $\inf _{x \in P \cap \partial \Omega_{2}} \rho(x)>0$ with $R / \inf _{x \in P \cap \partial \Omega_{2}} \rho(x) \leq 1 / k, \rho(A x) \geq \rho(x)$ and $A x \neq \mu x, \mu \in$ $(0,1), \forall x \in P \cap \partial \Omega_{2}$

hold, then $A$ has at least one fixed point in $P \cap\left(\Omega_{2} \backslash \bar{\Omega}_{1}\right)$.

Proof. It follows by taking $k+\delta=1$.

Corollary 2.9. Let $\Omega_{1}=\{x \in E \mid\|x\|<R\}$ and $\Omega_{2}$ a bounded open set in $E$ such that $\bar{\Omega}_{1} \subset \Omega_{2}$. Suppose that $A: P \cap\left(\bar{\Omega}_{2} \backslash \Omega_{1}\right) \rightarrow P$ is a k-set contraction with $k<1$ and $\rho: P \rightarrow[0, \infty)$ is a uniformly continuous convex functional with $\rho(\theta)=0$ and $\rho(x)>0, \forall x \neq \theta$ and $\rho(x) \leq\|x\|$. If

(a) $\inf _{x \in P \cap \partial \Omega_{1}} \rho(x)>0$ with $R / \inf _{x \in P \cap \partial \Omega_{1}} \rho(x) \leq 1 / k, \rho(A x) \geq \rho(x)$, and $A x \neq \mu x, \mu \in$ $(0,1], \forall x \in P \cap \partial \Omega_{1}$;

(b) $\rho(A x) \leq \rho(x), \forall x \in P \cap \partial \Omega_{2}$

hold, then $A$ has at least one fixed point in $P \cap\left(\Omega_{2} \backslash \bar{\Omega}_{1}\right)$.

Proof. It follows by taking $k+\delta=1$.

\section{Applications}

Throughout the remainder of this paper, we apply the above results to a second-order differential equation in Banach spaces:

$$
x^{\prime \prime}+f(t, x)=\theta, \quad 0<t<1,
$$

subject to the following integral boundary conditions:

$$
x(0)=\int_{0}^{1} g(t) x(t) d t, \quad x(1)=\theta,
$$

where $f \in C([0,1] \times P, P), \theta$ is the zero element of $E$, and $g \in L^{1}[0,1]$ is nonnegative.

We consider problem (3.1)-(3.2) in $C(J, E)$, in which $J=[0,1]$. Evidently, $\left(C(J, E),\|\cdot\|_{c}\right)$ is a Banach space with norm $\|x\|_{c}=\max _{t \in J}\|x(t)\|$ for $x \in C(J, E)$.

To establish the existence of positive solutions in $C(J, E)$ of (3.1)-(3.2), let us list the following assumptions.

$(\mathbb{H}) f \in C(J \times P, P)$, and for any $l>0, f$ is uniformly continuous on $J \times\left(P \cap \tau_{l}\right)$. Further suppose that $g \in L^{1}[0,1]$ is nonnegative, $\sigma \in[0,1)$, and there exist nonnegative constants $\eta_{l}$ with

$$
\frac{1}{2} \gamma \eta_{l}<1
$$


such that

$$
\alpha(f(t, S)) \leq \eta_{l} \alpha(S), \quad t \in J, S \subset P \cap \tau_{l},
$$

where $\tau_{l}=\{x \in E:\|x\| \leq l\}, \gamma=\left(1+\int_{0}^{1} s g(s) d s\right) /(1-\sigma)$, and $\sigma=\int_{0}^{1}(1-s) g(s) d s$.

It is easy to see that the problem (3.1)-(3.2) has a solution $x=x(t)$ if and only if $x$ is a solution of the operator equation

$$
(T x)(t)=\int_{0}^{1} H(t, s) f(s, x(s)) d s,
$$

where

$$
\begin{gathered}
H(t, s)=G(t, s)+\frac{1-t}{1-\sigma} \int_{0}^{1} G(s, \tau) g(\tau) d \tau, \\
G(t, s)= \begin{cases}t(1-s), & 0 \leq t \leq s \leq 1, \\
s(1-t), & 0 \leq s \leq t \leq 1 .\end{cases}
\end{gathered}
$$

From (3.6) and (3.7), we can prove that $H(t, s), G(t, s)$ have the following properties.

Proposition 3.1. Assume that $(\mathbb{H})$ holds. Then for $t, s \in[0,1]$ we have

$$
H(t, s) \geq 0, \quad G(t, s) \geq 0 .
$$

Proposition 3.2. For $t, s \in[0,1]$, we have

$$
e(t) e(s) \leq G(t, s) \leq G(t, t)=t(1-t)=e(t) \leq \bar{e}=\max _{t \in[0,1]} e(t)=\frac{1}{4}
$$

Proposition 3.3. Let $\bar{\delta} \in(0,1 / 2), J_{\bar{\delta}}=[\bar{\delta}, 1-\bar{\delta}]$. Then for all $t \in J_{\bar{\delta}}, s, u \in[0,1]$, we have

$$
G(t, s) \geq \bar{\delta} G(u, s)
$$

Proposition 3.4. Assume that $(\mathbb{H})$ holds. Then for $t, s \in[0,1]$, we have

$$
\rho e(t) e(s) \leq H(t, s) \leq \gamma t(1-t)=\gamma e(t),
$$


where $\gamma$ is defined in $(\mathbb{H})$, and

$$
\rho=\frac{\int_{0}^{1} e(\tau) g(\tau) d \tau}{1-\sigma}
$$

Proof. By (3.6) and (3.9), we have

$$
\begin{aligned}
H(t, s) & =G(t, s)+\frac{1-t}{1-\sigma} \int_{0}^{1} G(s, \tau) g(\tau) d \tau \\
& \geq \frac{1-t}{1-\sigma} \int_{0}^{1} G(s, \tau) g(\tau) d \tau \\
& \geq \frac{\int_{0}^{1} G(s, \tau) g(\tau) d \tau}{1-\sigma} t(1-t) \\
& \geq \frac{\int_{0}^{1} e(\tau) g(\tau) d \tau}{1-\sigma} t(1-t) s(1-s) \\
& =\rho e(t) e(s), \quad t \in[0,1] .
\end{aligned}
$$

On the other hand, noticing $G(t, s) \leq s(1-s)$, we obtain

$$
\begin{aligned}
H(t, s) & =G(t, s)+\frac{1-t}{1-\sigma} \int_{0}^{1} G(s, \tau) g(\tau) d \tau \\
& \leq s(1-s)+\frac{1-t}{1-\sigma} \int_{0}^{1} s(1-s) g(\tau) d \tau \\
& \leq s(1-s)\left[1+\frac{1}{1-\sigma} \int_{0}^{1} g(\tau) d \tau\right] \\
& \leq s(1-s) \frac{1+\int_{0}^{1} s g(s) d s}{1-\sigma} \\
& =r e(s), \quad t \in[0,1] .
\end{aligned}
$$

Proposition 3.5. Assume that $(\mathbb{H})$ holds. Then for all $t \in J_{\bar{\delta}}, s, u \in[0,1]$, we have

$$
H(t, s) \geq \bar{\delta} H(u, s)
$$


Proof. By (3.10), we have

$$
\begin{aligned}
H(t, s) & =G(t, s)+\frac{1-t}{1-\sigma} \int_{0}^{1} G(s, \tau) g(\tau) d \tau \\
& \geq \bar{\delta} G(u, s)+\frac{\bar{\delta}}{1-\sigma} \int_{0}^{1} G(s, \tau) g(\tau) d \tau \\
& \geq \bar{\delta} G(u, s)+\frac{\bar{\delta}(1-u)}{1-\sigma} \int_{0}^{1} G(s, \tau) g(\tau) d \tau \\
& =\bar{\delta} H(u, s), \quad s, u \in[0,1] .
\end{aligned}
$$

We construct a cone $K$ by

$$
K=\left\{x \in Q: x(t) \geq \bar{\delta} x(s), t \in J_{\bar{\delta}^{\prime}}, s \in[0,1]\right\}
$$

where

$$
Q=\{x \in C(J, E): x(t) \geq \theta, t \in J\} .
$$

It is easy to see that $K$ is a cone of $C(J, E)$.

We will make use of the following lemmas.

Lemma 3.6. Suppose that $(\mathbb{H})$ holds. Then for each $l>0, T$ is strict set contraction on $Q \cap B_{l}$, that is, there exists a constant $0 \leq k_{l}<1$ such that $\alpha(T(S)) \leq k_{l} \alpha(S)$ for any $S \subset Q \cap B_{l}$, where $B_{l}=\left\{x \in C[J, E],\|x\|_{c} \leq l\right\}$.

Proof. By $(\mathbb{H})$, we know that $f$ is uniformly continuous on $J \times\left(P \cap \tau_{l}\right)$. Hence, $f$ is bounded on $J \times\left(P \cap \tau_{l}\right)$. This together with (3.4) and Lemma 1.7 implies that

$$
\alpha(f(J \times S))=\max _{t \in J} \alpha(f(t, S)) \leq \eta_{l} \alpha(S), \quad \text { for } S \subset Q \cap B_{l} .
$$

From $f$ being uniformly continuous and bounded on $S \subset Q \cap B_{l}$, we can obtain that $T$ is continuous and bounded from $Q \cap B_{l}$ into $Q$.

On the other hand, it is clear that $0 \leq H(t, s) \leq(1 / 4) \gamma$ and using a similar method as in the proof of Lemma 2 in [40], we can get that

$$
\alpha(T(S)) \leq 2 \frac{1}{4} \gamma \eta_{l} \alpha(S)
$$

Therefore,

$$
\alpha(T(S)) \leq k_{l} \alpha(S), \quad S \subset Q \cap B_{l}
$$

where $k_{l}=(1 / 2) \gamma \eta_{l}, 0 \leq k_{l}<1$. The proof is complete. 
Lemma 3.7. Suppose that $(\mathbb{H})$ holds. Then $T(K) \subset K$ and $T: K \rightarrow K$ is a strict set contraction.

Proof. From (3.5) and (3.15), we obtain

$$
\begin{aligned}
\min _{t \in J_{\bar{\delta}}}(T x)(t) & =\min _{t \in J_{\bar{\delta}}} \int_{0}^{1} H(t, s) f(s, x(s)) d s \\
& \geq \bar{\delta} \int_{0}^{1} H(u, s) f(s, x(s)) d s \\
& \geq \bar{\delta}(T x)(u), \quad u \in J .
\end{aligned}
$$

Therefore, $T(x) \in K$, that is, $T(K) \subset K$.

Next by Lemma 3.6, one can prove that $T: K \rightarrow K$ is a strict set contraction. So it is omitted.

Let

$$
\Delta=\int_{\bar{\delta}}^{1-\bar{\delta}} H\left(\frac{1}{2}, s\right) d s .
$$

Theorem 3.8. Assume that $(\mathbb{H})$ holds and $P$ is normal. If there exist $a, b$ with $0<a<b$ such that $\Psi(f(t, x)) \geq \Delta^{-1} a$ for $t \in J_{\bar{\delta}},\|x\| \leq a$, and $\|f(t, x)\| \leq 6 \gamma^{-1} b$ for $t \in J_{\bar{\delta}},\|x\| \leq \bar{\delta}^{-1} b$, where $\Psi \in P^{*},\|\Psi\|=1$, then problem (3.1)-(3.2) has at least one positive solution.

Proof. Let $T$ be the cone preserving, strict set contraction that was defined by (3.5).

Let $\rho(x)=\sup _{t \in J_{\bar{\sigma}}}\|x(t)\|$. Then $\rho: K \rightarrow[0,+\infty)$ is a uniformly continuous convex functional with $\rho(\theta)=0$ and $\rho(x)>0$ for $x \neq \theta$. Let

$$
\Omega_{1}=\left\{x \in C[J, E] \mid\|x\|_{C}<a\right\}, \quad \Omega_{2}=\{x \in C[J, E] \mid \rho(x)<b\} .
$$

It is clear that $\Omega_{1}$ and $\Omega_{2}$ are open sets in $C[J, E]$ with $\theta \in \Omega_{1}$ and $\bar{\Omega}_{1} \subset \Omega_{2}$. If $x \in K \cap \Omega_{2}$, we have $\|x\|_{c} \leq \bar{\delta}^{-1} b$ which implies that $K \cap \Omega_{2}$ is bounded.

If $x \in K \cap \partial \Omega_{1}$, then $\|x\|_{c}=a$.

$$
\begin{aligned}
\rho(T x) & =\sup _{t \in J_{\bar{\delta}}}\|(T x)(t)\| \geq\left\|(T x)\left(\frac{1}{2}\right)\right\| \\
& \geq \Psi\left((T x)\left(\frac{1}{2}\right)\right)=\int_{0}^{1} H\left(\frac{1}{2}, s\right) \Psi(f(s, x(s))) d s \\
& \geq \int_{\bar{\delta}}^{1-\bar{\delta}} H\left(\frac{1}{2}, s\right) \Psi(f(s, x(s))) d s \\
& \geq \int_{\bar{\delta}}^{1-\bar{\delta}} H\left(\frac{1}{2}, s\right) d s \Delta^{-1} a=a=\|x\|_{c} \geq \rho(x) .
\end{aligned}
$$




$$
\begin{aligned}
& \text { If } x \in K \cap \partial \Omega_{2} \text {, then } \rho(x)=b \text { and }\|x\|_{c} \leq \bar{\delta}^{-1} b . \\
& \rho(T x)=\sup _{t \in J_{\bar{\delta}}}\|(T x)(t)\| \leq \sup _{t \in J_{\bar{\delta}}} \int_{0}^{1} H(t, s)\|f(s, x(s))\| d s \leq 6 \gamma^{-1} b \sup _{t \in J_{\bar{\delta}}} \int_{0}^{1} H(t, s) d s=b=\rho(x) .
\end{aligned}
$$

Hence, the proof is finished by Corollary 2.9.

\section{Example}

Example 4.1. To illustrate how our main results can be used in practice, we present an example. For the convenience of computation, we study a two-point boundary value problem. Now we consider the following boundary value problem:

$$
\begin{gathered}
-x^{\prime \prime}=f(t, x), \quad 0<t<1, \\
x(0)=0, \quad x(1)=0,
\end{gathered}
$$

where $g(t)=0$, and

$$
f(t, x)= \begin{cases}10 t e^{-x}+25, & 0<t<1,0 \leq x \leq 10 \\ \frac{1}{324}(x-100)^{2}+10 t e^{-10}, & 0<t<1, x \geq 10\end{cases}
$$

Hence $\sigma=0, \gamma=1$. In this case $E=R, P=R^{+}$, and $k=0$. Let $\Psi \equiv 1, \bar{\delta}=1 / 10$; then $\Delta=2 / 5$.

Select $a=10, b=20$; then we can see that

$$
\begin{gathered}
|f(t, x)| \geq 10 \times \frac{1}{10} \times e^{-10}+25>25=\Delta^{-1} a, \quad \forall t \in\left[\frac{1}{10}, \frac{9}{10}\right], x \leq 10 \\
|f(t, x)| \leq \frac{1}{324} \times 100^{2}+10 \times \frac{1}{10} \times e^{-10}<60=6 \gamma^{-1} a, \quad \forall t \in\left[\frac{1}{10}, \frac{9}{10}\right], x \leq 200 .
\end{gathered}
$$

Hence, the conditions of the Theorem 3.8 are satisfied. Then problem (4.1) has at least one positive solution.

Remark 4.2. Example 4.1 implies that there is a large number of functions that satisfy the conditions of Theorem 3.8. In addition, the conditions of Theorem 3.8 are also easy to check.

\section{Acknowledgments}

This work is sponsored by the Funding Project for Academic Human Resources Development in Institutions of Higher Learning Under the Jurisdiction of Beijing Municipality (PHR201008430), the Scientific Research Common Program of Beijing Municipal Commission of Education (KM201010772018), the 2010 level of scientific research of improving project 
(5028123900), the Level of Graduate Education of Improving-Graduate Technology Innovation Project (5028211000), and Beijing Municipal Education Commission (71D0911003). The authors thank the referee for his/her careful reading of the manuscript and useful suggestions.

\section{References}

[1] D. Guo, V. Lakshmikantham, and X. Liu, Nonlinear Integral Equations in Abstract Spaces, vol. 373 of Mathematics and Its Applications, Kluwer Academic Publishers, Dordrecht, The Netherlands, 1996.

[2] D. Guo and V. Lakshmikantham, Nonlinear Problems in Abstract Cones, vol. 5 of Notes and Reports in Mathematics in Science and Engineering, Academic Press, Boston, Mass, USA, 1988.

[3] V. Lakshmikanthan and S. Leela, Nonlinear Differential Equations in Abstract Spaces, Pergamon, Oxford, UK, 1981.

[4] K. Demling, Ordinary Differential Equations in Banach Spaces, Springer, Berlin, Germany, 1977.

[5] M. Feng and H. Pang, "A class of three-point boundary-value problems for second-order impulsive integro-differential equations in Banach spaces," Nonlinear Analysis: Theory, Methods \& Applications, vol. 70, no. 1, pp. 64-82, 2009.

[6] M. Feng, D. Ji, and W. Ge, "Positive solutions for a class of boundary-value problem with integral boundary conditions in Banach spaces," Journal of Computational and Applied Mathematics, vol. 222, no. 2, pp. 351-363, 2008.

[7] D. Guo, "Multiple positive solutions for first order nonlinear impulsive integro-differential equations in a Banach space," Applied Mathematics and Computation, vol. 143, no. 2-3, pp. 233-249, 2003.

[8] D. Guo, "Multiple positive solutions of a boundary value problem for $n$ th-order impulsive integrodifferential equations in a Banach space," Nonlinear Analysis: Theory, Methods \& Applications, vol. 56, no. 7, pp. 985-1006, 2004.

[9] D. Guo, "Multiple positive solutions for $n$ th-order impulsive integro-differential equations in Banach spaces," Nonlinear Analysis: Theory, Methods E Applications, vol. 60, no. 5, pp. 955-976, 2005.

[10] Y. Cui and Y. Zou, "Positive solutions of nonlinear singular boundary value problems in abstract spaces," Nonlinear Analysis: Theory, Methods E Applications, vol. 69, no. 1, pp. 287-294, 2008.

[11] X. Zhang and L. Liu, "Initial value problems for nonlinear second order impulsive integro-differential equations of mixed type in Banach spaces," Nonlinear Analysis: Theory, Methods \& Applications, vol. 64, no. 11, pp. 2562-2574, 2006.

[12] L. Liu, Z. Liu, and Y. Wu, "Infinite boundary value problems for $n$ th-order nonlinear impulsive integro-differential equations in Banach spaces," Nonlinear Analysis: Theory, Methods \& Applications, vol. 67, no. 9, pp. 2670-2679, 2007.

[13] Y. Liu, "Boundary value problems for second order differential equations on unbounded domains in a Banach space," Applied Mathematics and Computation, vol. 135, no. 2-3, pp. 569-583, 2003.

[14] Y. Liu and A. Qi, "Positive solutions of nonlinear singular boundary value problem in abstract space," Computers $\mathcal{E}$ Mathematics with Applications, vol. 47, no. 4-5, pp. 683-688, 2004.

[15] B. Liu, "Positive solutions of a nonlinear four-point boundary value problems in Banach spaces," Journal of Mathematical Analysis and Applications, vol. 305, no. 1, pp. 253-276, 2005.

[16] X. Zhang, M. Feng, and W. Ge, "Existence and nonexistence of positive solutions for a class of $n$ thorder three-point boundary value problems in Banach spaces," Nonlinear Analysis: Theory, Methods $\mathcal{E}$ Applications, vol. 70, no. 2, pp. 584-597, 2009.

[17] X. Zhang, "Existence of positive solutions for multi-point boundary value problems on infinite intervals in Banach spaces," Applied Mathematics and Computation, vol. 206, no. 2, pp. 932-941, 2008.

[18] X. Zhang, "Existence of positive solution for second-order nonlinear impulsive singular differential equations of mixed type in Banach spaces," Nonlinear Analysis: Theory, Methods E Applications, vol. 70, no. 4, pp. 1620-1628, 2009.

[19] J. X. Sun, "A generalization of Guo's theorem and applications," Journal of Mathematical Analysis and Applications, vol. 126, no. 2, pp. 566-573, 1987.

[20] D. R. Anderson and R. I. Avery, "Fixed point theorem of cone expansion and compression of functional type," Journal of Difference Equations and Applications, vol. 8, no. 11, pp. 1073-1083, 2002.

[21] Y. Guo and W. Ge, "Positive solutions for three-point boundary value problems with dependence on the first order derivative," Journal of Mathematical Analysis and Applications, vol. 290, no. 1, pp. 291-301, 2004. 
[22] G. Zhang and J. Sun, "A generalization of the cone expansion and compression fixed point theorem and applications," Nonlinear Analysis: Theory, Methods $\mathcal{E}$ Applications, vol. 67, no. 2, pp. 579-586, 2007.

[23] J. M. Gallardo, "Second-order differential operators with integral boundary conditions and generation of analytic semigroups," The Rocky Mountain Journal of Mathematics, vol. 30, no. 4, pp. 1265-1292, 2000.

[24] G. L. Karakostas and P. Ch. Tsamatos, "Multiple positive solutions of some Fredholm integral equations arisen from nonlocal boundary-value problems," Electronic Journal of Differential Equations, vol. 2002, no. 30, pp. 1-17, 2002.

[25] A. Lomtatidze and L. Malaguti, "On a nonlocal boundary value problem for second order nonlinear singular differential equations," Georgian Mathematical Journal, vol. 7, no. 1, pp. 133-154, 2000.

[26] B. Ahmad, A. Alsaedi, and B. S. Alghamdi, "Analytic approximation of solutions of the forced Duffing equation with integral boundary conditions," Nonlinear Analysis: Real World Applications, vol. 9, no. 4, pp. 1727-1740, 2008.

[27] M. Feng, B. Du, and W. Ge, “Impulsive boundary value problems with integral boundary conditions and one-dimensional p-Laplacian," Nonlinear Analysis: Theory, Methods \& Applications, vol. 70, no. 9, pp. 3119-3126, 2009.

[28] A. Boucherif, "Second-order boundary value problems with integral boundary conditions," Nonlinear Analysis: Theory, Methods \& Applications, vol. 70, no. 1, pp. 364-371, 2009.

[29] G. Infante and J. R. L. Webb, "Nonlinear non-local boundary-value problems and perturbed Hammerstein integral equations," Proceedings of the Edinburgh Mathematical Society, vol. 49, no. 3, pp. 637-656, 2006.

[30] P. Kang, Z. Wei, and J. Xu, "Positive solutions to fourth-order singular boundary value problems with integral boundary conditions in abstract spaces," Applied Mathematics and Computation, vol. 206, no. 1, pp. 245-256, 2008.

[31] H. Ma, "Symmetric positive solutions for nonlocal boundary value problems of fourth order," Nonlinear Analysis: Theory, Methods E Applications, vol. 68, no. 3, pp. 645-651, 2008.

[32] J. R. L. Webb, "Positive solutions of some three point boundary value problems via fixed point index theory," Nonlinear Analysis: Theory, Methods \& Applications, vol. 47, no. 7, pp. 4319-4332, 2001.

[33] J. R. L. Webb and G. Infante, "Positive solutions of nonlocal boundary value problems: a unified approach," Journal of the London Mathematical Society, vol. 74, no. 3, pp. 673-693, 2006.

[34] J. R. L. Webb, G. Infante, and D. Franco, "Positive solutions of nonlinear fourth-order boundary-value problems with local and non-local boundary conditions," Proceedings of the Royal Society of Edinburgh. Section A, vol. 138, no. 2, pp. 427-446, 2008.

[35] Z. Yang, "Positive solutions to a system of second-order nonlocal boundary value problems," Nonlinear Analysis: Theory, Methods E Applications, vol. 62, no. 7, pp. 1251-1265, 2005.

[36] X. Zhang, M. Feng, and W. Ge, "Existence results for nonlinear boundary-value problems with integral boundary conditions in Banach spaces," Nonlinear Analysis: Theory, Methods E Applications, vol. 69, no. 10, pp. 3310-3321, 2008.

[37] X. Zhang, M. Feng, and W. Ge, "Symmetric positive solutions for $p$-Laplacian fourth-order differential equations with integral boundary conditions," Journal of Computational and Applied Mathematics, vol. 222, no. 2, pp. 561-573, 2008.

[38] X. Zhang, M. Feng, and W. Ge, "Existence result of second-order differential equations with integral boundary conditions at resonance," Journal of Mathematical Analysis and Applications, vol. 353, no. 1, pp. 311-319, 2009.

[39] Y.-K. Chang, J. J. Nieto, and W.-S. Li, “On impulsive hyperbolic differential inclusions with nonlocal initial conditions," Journal of Optimization Theory and Applications, vol. 140, no. 3, pp. 431-442, 2009.

[40] D. Guo and V. Lakshmikantham, "Multiple solutions of two-point boundary value problems of ordinary differential equations in Banach spaces," Journal of Mathematical Analysis and Applications, vol. 129, no. 1, pp. 211-222, 1988. 


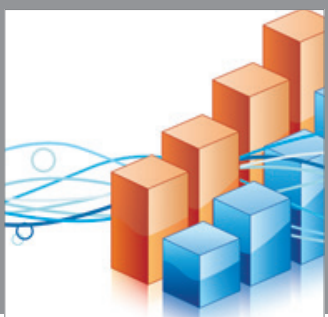

Advances in

Operations Research

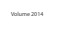

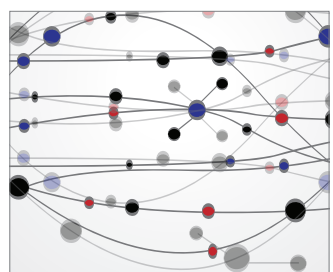

\section{The Scientific} World Journal
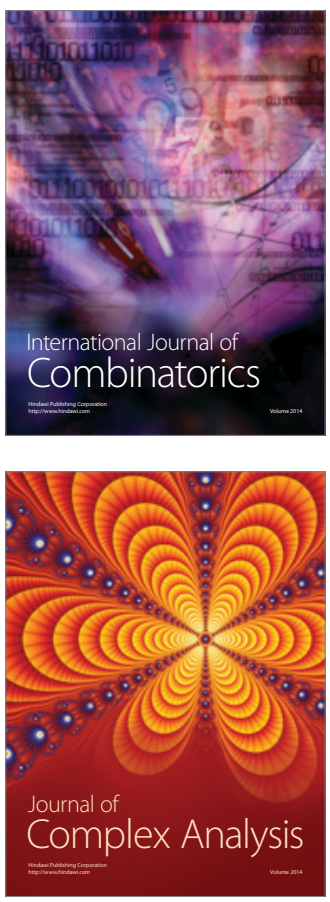

International Journal of

Mathematics and

Mathematical

Sciences
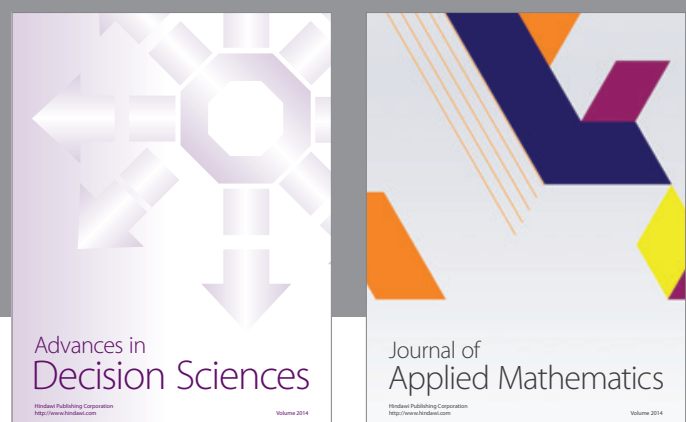

Journal of

Applied Mathematics
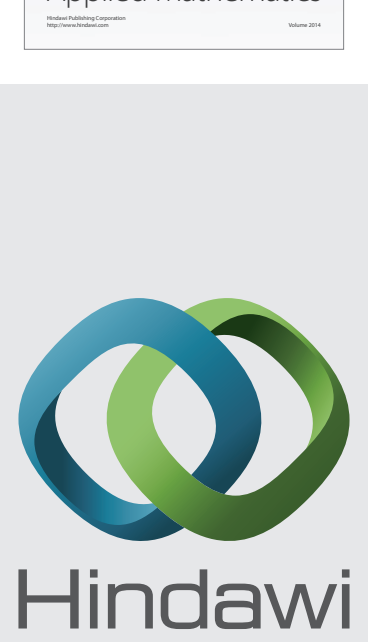

Submit your manuscripts at http://www.hindawi.com
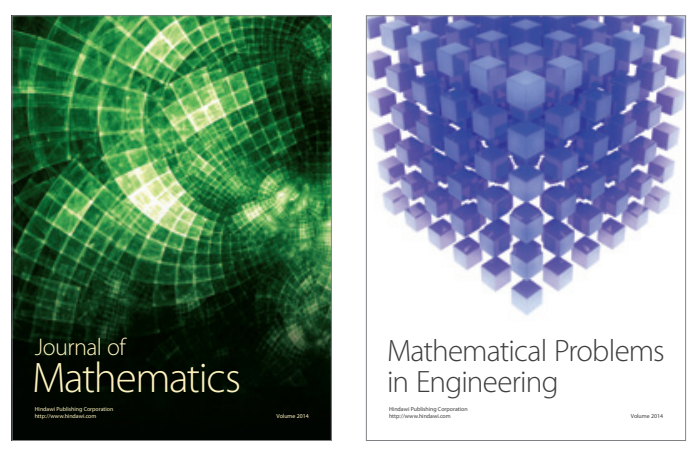

Mathematical Problems in Engineering
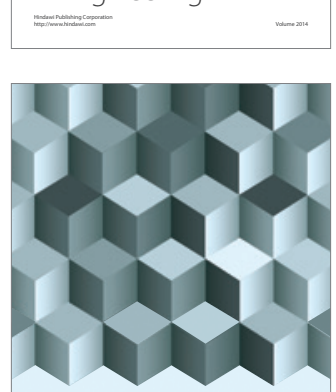

Journal of

Function Spaces
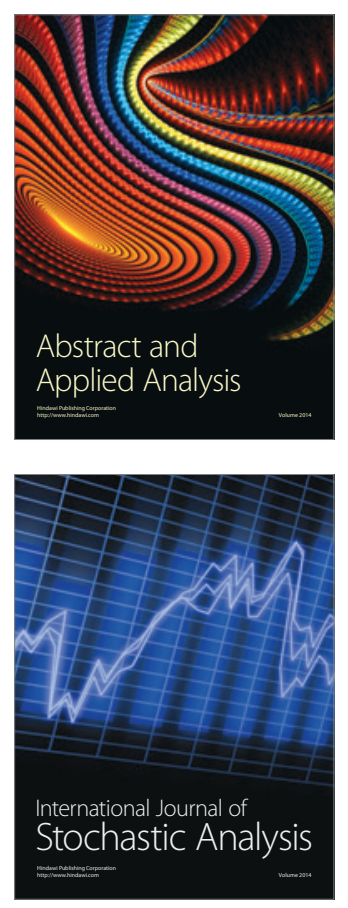

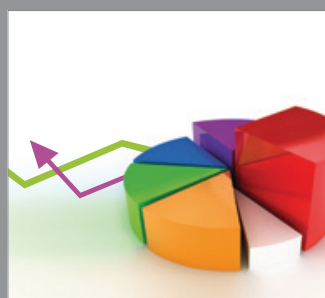

ournal of

Probability and Statistics

Promensencen
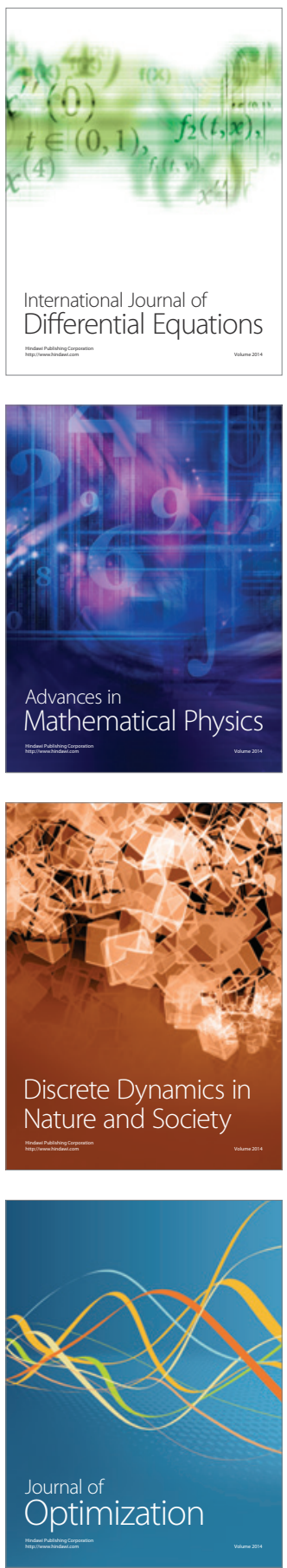